\title{
Correlation of Tumour-Infiltrating Lymphocyte Volume (TILV) with Histological Grading in Colorectal Adenocarcinoma Belman Novenry Silalahi ${ }^{\mathrm{a}}$, Betty ${ }^{\mathrm{b}}$, Delyuzar ${ }^{\mathrm{c}}$, T. Ibnu Alferraly ${ }^{\mathrm{d}}$, Jessy Chrestella ${ }^{\mathrm{e}}$
}

\author{
abelmansilalahi07@gmail.com \\ a Department of Anatomical Pathology, Faculty of Medicine, Universitas Sumatera Utara, Jalan Universitas No.1, Medan 20155, \\ Indonesia. \\ ${ }^{b}$ Department of Anatomical Pathology, Faculty of Medicine, Universitas Sumatera Utara, Jalan Universitas No.1, Medan 20155, \\ Indonesia.
}

\begin{abstract}
Background: Colorectal adenocarcinoma is a malignant epithelial tumour originating in the large bowel, showing glandular or mucinous differentiation. This tumour is the second most common cause of death due to cancer. The prognose can be predicted by evaluating the histological grading and tumour-infiltrating lymphocyte volume (TILV). TILV calculation is performed by multiplication of stromal TILs and tumour-stroma ratio (TSR). TILV score $\geq 1300$ have significantly better prognosis.

Methods: This is an analytical study with cross sectional approach on 32 resection specimens from the patients diagnosed as colorectal adenocarcinoma stained with Hematoxylin \& Eosin. The statistical analysis was performed by using Spearman rank correlation to analyse the association of TILV score and histological grading of colorectal adenocarcinoma.

Results: There were 32 sample of colorectal adenocarcinoma observed and the number of the low grade was greater than the high grade. Tumour with lower grade had higher stromal TILs and lower proportion of stroma. Colorectal adenocarcinoma with higher grade had lower TILV score. The TILV score showed significantly strong correlation with histological grading of colorectal adenocarcinoma.
\end{abstract}

Keywords: colorectal adenocarcinoma; stromal TIL; TSR; TILV

\section{INTRODUCTION}

Colorectal carcinoma (CRC) is a malignant epithelial tumour originating in the large bowel. Highest incidence was observed in population with highly animal fat consumption and sedentary lifestyle. ${ }^{1,2}$ This cancer is the second most common cause of death due to cancer in the world. According to Global Burden Cancer (GLOBOCAN), there are more than 1.8 million new cases and 881.000 deaths due to this carcinoma in 2018. There are $30.017(8,6 \%)$ new cases in 2018 and is the $4^{\text {th }}$ most common malignancy in Indonesia. ${ }^{3,4}$ In H. Adam Malik General Hospital, the incidence of this cancer increased from 2015 until 2017. ${ }^{5}$

Grading of CRC is based on gland formation; low and high grade. Low grade CRC is formerly well- to moderately differentiated and high-grade is formerly poorly differentiated tumours. ${ }^{1}$ The prognosis of this tumour can be predicted by host immune response to cancer that is lymphocytes infiltration in tumour cells or TILs (tumour-infiltrating lymphocytes). Many studies revealed stromal TILs has more significant prognostic value than intratumoural TILs. ${ }^{6,7}$

However, the current TILs evaluation system does not include the stromal ratio and may not adequately reflect the true capacity of tumour immunity. Fard et al and Zhang et al. introduced the tumour-infiltrating lymphocyte volume (TILV) by considering both the tumour stromal volume and the stromal TILs to precisely evaluate the capacity of tumour immunity in TNBC..$^{8,9}$

TILV assessment is still infrequently performed especially in CRC. This is the main reason of this study to 
evaluate TILV in CRC and to analyse the correlation of tumour-infiltrating lymphocyte volume (TILV) with its histological grading.

\section{MATERIALS AND METHODS}

In this study, we evaluated 32 samples of CRC from surgical specimen in H. Adam Malik General Hospital, Medan. All samples were histopathologically diagnosed as adenocarcinoma colorectal by Hematoxylin \& Eosin staining and reviewed by two pathologists in Department of Anatomical Pathology, Faculty of Medicine, Universitas Sumatera Utara, Medan.

TILV calculation was performed by multiplication of stromal TILs and tumour-stroma ratio (TSR). Stromal TILs Stromal TILs were assessed according to the standardization and guidelines of the international TILs working group. Two predefined categories were used-poor TILs $(<60 \%)$ and rich TILs $(\geq 60 \%)$-for the statistical analysis. $6,8,9$ TSR was assessed by a lower-power $(40 \times)$ evaluation for the tumour stroma percentage. The cutoff point for TSR was $50 \%$; stroma percentage $<50 \%$ was categorized as stroma poor or TSR-high and $\geq 50 \%$ as stroma rich or TSR;low. 8,910

A tumour had $20 \%$ tumour stroma and $70 \%$ stromal TILs; the TILV $=20 \times 70=1400$. Another tumour had 50\% tumour stroma and $5 \%$ stromal TILs; the TILV $=50 \times 5=250$. The TILV value 1300 was used as the cut off point; TILV $\geq 1300$ was considered as TILV high and TILV $<1300$ as a TILV low. ${ }^{8,9}$ The statistical analysis was performed by using Spearman rank correlation to analyse the association of TILV score and histological grading of colorectal adenocarcinoma.

\section{RESULTS}

There were 32 samples of CRC observed; 21 samples $(65,63 \%)$ of low grade CRC and 11 samples $(34,37 \%)$ of high grade CRC. Stromal TILs percentage was examined by evaluating the infiltration of lymphocytes in stroma of the tumour by 200x magnification. We obtained 13 samples of TILs rich $(40,63 \%)$ and 19 samples of TILs poor $(59,37 \%)$. The majority tumour stroma was stroma poor or TSR-high (13 samples or $59,37 \%)$. There were 13 samples $(40,63 \%)$ of stroma rich (TSR-low).

The most novel finding in this study was the concept of TILV by incorporating both the tumourstromal ratio and stromal TILs to assess the immune response. Our study obtained 21 samples of TILV high $(65,63 \%)$ and 11 samples of TILV low $(34,37 \%)$, and therefore, the majority TILV value was TILV high.

The distribution of correlation between TILV value and grading of colorectal adenocarcinoma including their correlation test can be seen in table 1 and table 2 .

Table 1. Distribution of correlation between TIL value and grading of colorectal adenocarcinoma.

\begin{tabular}{|c|c|c|c|c|c|c|}
\hline \multirow{3}{*}{ No. } & \multirow{3}{*}{$\begin{array}{l}\text { Tumour-Infiltrating } \\
\text { Lymphocyte Volume } \\
\text { (TILV) }\end{array}$} & \multicolumn{4}{|c|}{ Histological Grading } & \multirow{3}{*}{ Total } \\
\hline & & \multicolumn{3}{|c|}{ Low grade } & High grade & \\
\hline & & $\mathrm{n}$ & $\%$ & $\mathrm{~N}$ & $\%$ & \\
\hline 1. & High & 21 & 100 & 0 & 0 & \\
\hline 2. & Low & 0 & 0 & 11 & 100 & \\
\hline & Total & 21 & & 11 & & 32 \\
\hline
\end{tabular}


Table 2. Correlation test between TILV value and histological grading of colorectal adenocarcinoma.

\begin{tabular}{cccc}
\hline \multirow{2}{*}{ Variable } & \multicolumn{3}{c}{ Histological Grading } \\
\cline { 2 - 4 } & $\mathrm{N}$ & $\mathrm{r}$ & $\mathrm{p}$ \\
\hline TILV & 32 & $-0,828$ & 0,0001 \\
\hline
\end{tabular}

Spearman's rank test revealed the correlation between TILV value and histological grading of CRC was significant with $\mathrm{p}$ value $=0,0001(\mathrm{p}<0,05)$. The Spearman's rank correlation coefficient was very strong $(r=0,828)$ and the direction of the relationship was negative which defined that the higher the TILV value, the lower is the grading of $\mathrm{CRC}$, and vice versa.
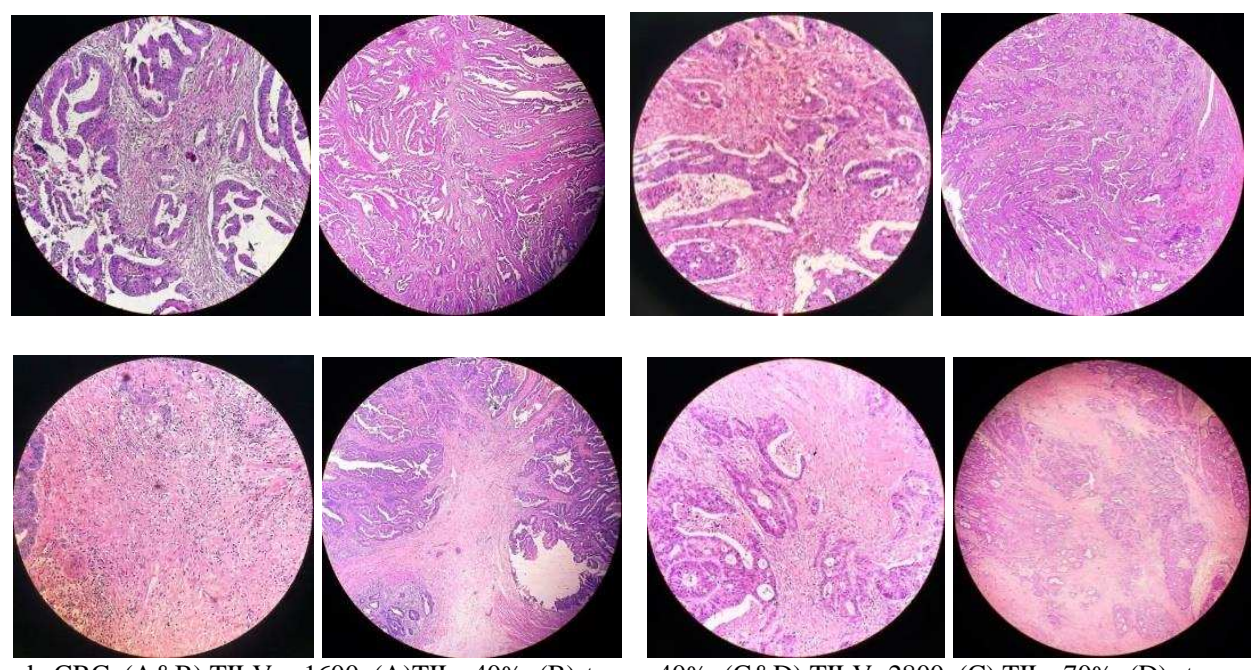

Fig1. Low grade CRC. (A\&B) TILV = 1600, (A)TILs 40\%, (B)stroma 40\%, (C\&D) TILV=2800, (C) TILs 70\%, (D) stroma 40\%, (E\&F) TILV=5600, (E) TILs 80\%, (F) stroma 70\%, (G\&H) TILV=2100, (G) TILs 30\%, (H) stroma 70\%. (H\&E)
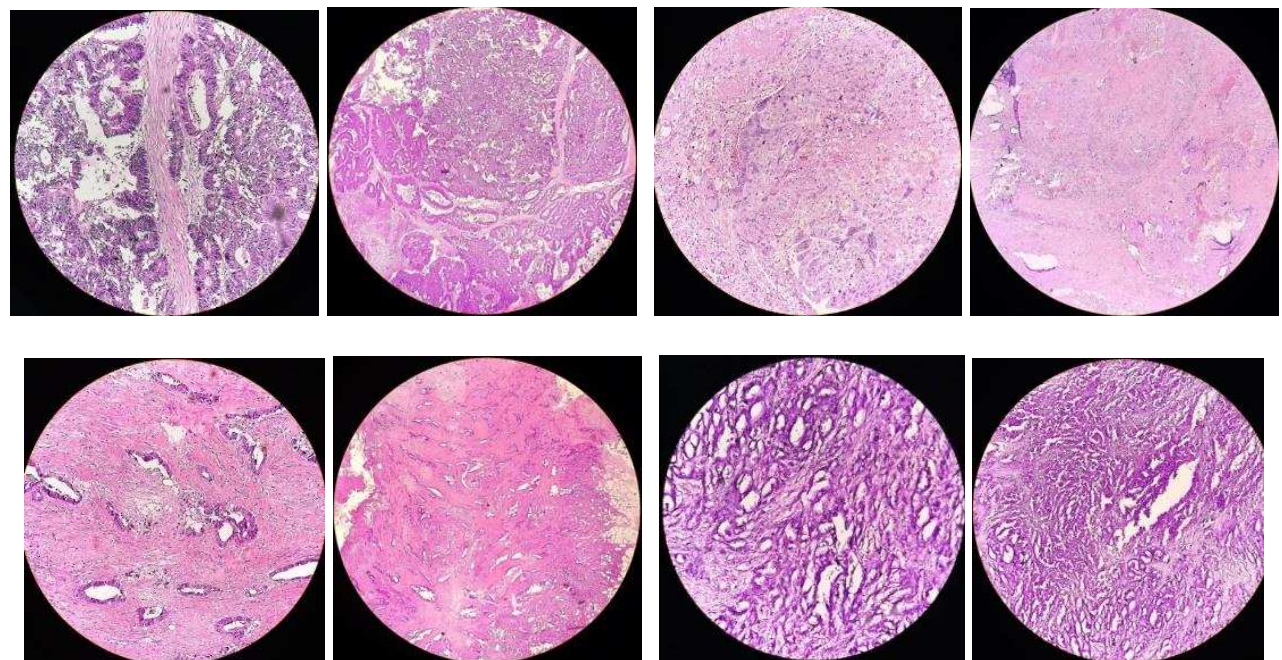

Fig 2. High grade CRC. (A\&B) TILV= 200 (A) TILs 10\%, (B) stroma 20\%, (C\&D) TILV=1200, (C) TILs 20\%, (D) stroma 60\%, (E\&F) TILV=700, (E) TILs 10\%, (F) stroma 70\%, (G\&H) TILV=300, (G) TILs 10\%, (H) stroma 30\%. (H\&E) 


\section{DISCUSSION}

There were 32 specimens of CRC in this study with low grade CRC was the greatest number $(65,63 \%)$ while high grade CRC was 34,37\%. Recently, the studies of Schwarz et al. and Zlobec et al. in 2020 had the low grade CRC as the greatest number. The previous study by Nasution et al. in H. Adam Malik General Hospital from 2015 until 2017 giving the similar result, well differentiated CRC was 44,4\%, moderately differentiated CRC was $35,8 \%$ and poorly differentiated was $19,8 \% .{ }^{5}$ Histological grading can be used as a predictor for prognosis in CRC. the higher the grading of CRC, the worse is the prognosis.

In this study, we found that more than 50\% $(61,9 \%)$ of low grade CRC had TILS rich while all (100\%) of high grade CRC had TILS poor. This finding is in accordance with the study of Nasution et al in 2020. They found more TILS rich in well differentiated CRC compared to TILS poor. ${ }^{6}$ Jakubowska et al defined that the increasing of grading is always followed by the decreasing of TILS value. We found that the majority stroma of low grade CRC was stroma poor $(61,9 \%)$ while stroma in high grade CRC was almost the same between stroma poor and stroma rich $(54,5 \%$ vs $45,5 \%)$. This finding is in accordance with the study of Hilda et al in 2019. they found the majority stroma in well differentiated CRC was stroma poor (TSR high). ${ }^{10}$ It can be concluded that the lower grade of CRC has a little amount of stroma and vice versa. All low grade CRC (100\%) in this study had TILV high while all high grade CRC (100\%) had TILV low. There was a significant correlation $(\mathrm{p}=0,0001)$ between histological grading of $\mathrm{CRC}$ and TILV value. Spearman's rank test revealed that histological grading was strongly associated with TILV value $(r=0,828) .{ }^{11}$ The negative direction of coefficient showed a reverse relationship. The higher is the grading, the lower is TILV value and vice versa.

There was no study before to evaluate the correlation between TILV value and histological grading in CRC. Same as TILS, TILV value also reflects tumour immunity but the difference is when evaluating TILV value, stromal volume is also considered so that this value indicate the real capacity of tumour immunity and in addition, TILV value correlates with prognosis. Fard et al. and Zhang et al. reported that high TILV score $(\geq 1300)$ showed excellent overall survival rate and disease-free survival rate in TNBC. ${ }^{8,9}$ This concept can be applied to CRC since they are similar, both of them are solid tumour. We also adopted their guideline in evaluating TILV value to our study.

\section{CONCLUSION}

The histological grading of CRC was very strongly associated with TILV value and the direction showed a reverse relationship. The higher is the histological grading, the lower is TILV value. TILV value is easy to evaluate and adds additional dimension to TILs. This parameter can be directly reported in daily practice when diagnosing CRC. Recent study reported that TILV value can predict the therapeutic efficacy and can be used as a predictor for prognosis.

\section{THANK YOU}

We would like to thank to The Department of Anatomical Pathology, Universitas Sumatera Utara and H. Adam Malik General Hospital Medan, Indonesia for their contribution,

\section{ETHICAL APPROVAL}

Health Research Ethical Committee. Universitas Sumatera Utara Medan, Indonesia had approved this study.

\section{ACKNOWLEDGESMENTS}

This study originated as an assignment in The Department of Anatomical Pathology, Universitas Sumatera Utara. The authors would thus like to deeply thank to all who had contribution in this study. 


\section{References}

Nagtegaal ID, Arends MJ, Salto-Tellez M. Colorectal adenocarcinoma. In WHO Classification of Tumours: Digestive System Tumours. $5^{\text {th }}$. WHO Classification of Tumours Editorial Board (Ed), International Agency for Research on Cancer. Lyon 2019. Pp.177-87.

Bray F, Ferlay J, SoerjomataramI, Siegel RL, Torre LA, Jemal A. Global Cancer Statistics. CA Cancer Journal for Clinicians. 2018. Pp.394-424.

International Agency for Research on Cancer. Asia, Source : Globocan 2018 . Available from : http://gco.iarc.fr/today/data/factsheets/populations/935-asia-fact-sheets.pdf.

International Agency for Research on Cancer. Indonesia, Source : Globocan 2018. Available from : http://gco.iarc.fr/today/data/factsheets/populations/360-indonesia-fact-sheets.pdf

Nasution, Novia. 2018. Karakteristik Pasien Kanker Kolorektal di RSUP H. Adam Malik Medan 2015-2017. [ cited 2019 apr 24]. Available from : http://repositori.usu.ac.id/handle/123456789/11025.

Nasution FI, Lubis ND, Betty. Correlation of intratumoral tumor-infiltrating lymphocytes (TILs) with histological grading of adenocarcinoma colon. International Journal of Research Publications. 2020; 45:1-7.

Jakubowska K, Kisielewski W, Kańczuga-KL, Koda M, Famulski W. Stromal and intraepithelial tumor-infiltrating lymphocytes in colorectal carcinoma. Oncol Lett. 2017; 14(6): 6421-32.

Hilda S, Soekimin, Betty. Correlation of tumor-stroma ratio (TSR) with histological grading and staging in colorectal adenocarcinoma. International Journal of Research Publications. 2020;45:1-9.

Fard EV, Ali Y, Wang XI, Saluja K, Covinsky MH, Wang L, et al. Tumor infiltrating lymphocyte volume is a better predictor of disease free survival than stromal tumor infiltrating lymphocytes in invasive breast carcinoma. American Journal Clinical Pathology. 2019; $20: 1-10$.

Zhang L, Wang XI, Zhang S. Tumor infiltrating lymphocyte volume is a better predictor of neoadjuvant therapy response and overall survival in triple negative invasive breast cancer. Human Pathology. Elsevier. 2018; 80:47-54.

Sugiyono. Metode penelitian kuantitatif, kualitatif dan R\&D. Alfabeta, Bandung. 2010. Pp.149-53. 